\title{
Structural analysis of FAD synthetase from Corynebacterium ammoniagenes
}

\author{
Susana Frago, Marta Martínez-Júlvez, Ana Serrano and Milagros Medina*
}

Address: Departamento de Bioquímica y Biología Molecular y Celular, Facultad de Ciencias and Institute of Biocomputation and Physics of Complex Systems, Universidad de Zaragoza, Zaragoza, Spain

Email: Susana Frago - sfrago@unizar.es; Marta Martínez-Júlvez - mmartine@unizar.es; Ana Serrano - anaserra@unizar.es; Milagros Medina* - mmedina@unizar.es

* Corresponding author

Published: 23 September 2008

BMC Microbiology 2008, 8:160 doi:10.1/86/147/-2180-8-160
Received: 7 May 2008

Accepted: 23 September 2008

This article is available from: http://www.biomedcentral.com/I47I-2/80/8/I60

(C) 2008 Frago et al; licensee BioMed Central Ltd.

This is an Open Access article distributed under the terms of the Creative Commons Attribution License (http://creativecommons.org/licenses/by/2.0), which permits unrestricted use, distribution, and reproduction in any medium, provided the original work is properly cited.

\begin{abstract}
Background: The prokaryotic FAD synthetase family - a group of bifunctional enzymes that catalyse riboflavin phosphorylation and FMN adenylylation within a single polypeptide chain- was analysed in terms of sequence and structure.

Results: Sequences of nearly 800 prokaryotic species were aligned. Those related with bifunctional FAD synthetase activities showed conservation of several consensus regions and highly conserved residues. A 3D model for the FAD synthetase from Corynebacterium ammoniagenes (CaFADS) was generated. This model confirms that the $\mathrm{N}$-terminal and $\mathrm{C}$-terminal domains are related to nucleotydyltransferases and riboflavin kinases, respectively. Models for the interaction of CaFADS with its substrates were also produced, allowing location of all the protein substrates in their putative binding pockets. These include two independent flavin binding sites for each CaFADS activity.

Conclusion: For the first time, the putative presence of a flavin binding site for the adenylylation activity, independent from that related with the phosphorylation activity, is shown. Additionally, these models suggest the functional relevance of some residues putatively involved in the catalytic processes. Their relevant roles were analysed by site-directed mutagenesis. A role was confirmed for $\mathrm{H} 28, \mathrm{H} 3 \mathrm{I}, \mathrm{SI} 64$ and TI65 in the stabilisation of the P groups and the adenine moiety of ATP and, the P of FMN for the adenylylation. Similarly, T208, N210 and E268 appear critical for accommodation of the $\mathrm{P}$ groups of ATP and the ribityl end of RF in the active site for the phosphorylation process. Finally, the C-terminal domain was shown to catalyse the phosphorylation process on its own, but no reaction at all was observed with the individually expressed $\mathrm{N}$-terminal domain.
\end{abstract}

\section{Background}

The activities of riboflavin (RF) phosphorylation and FMN adenylylation are present among all kingdoms of living organisms. However, whereas eukaryotes use two different enzymes for FMN (flavokinase) and FAD (ade- nylyltransferase) production [1-6], prokaryotic organisms depend on an enzyme of $~ 38 \mathrm{kDa}$, FAD synthetase (Flavin adenine dinucleotide synthetase, FADS), that exhibits both activities [7]. Thus, FADS constitutes a bifunctional prokaryotic protein family of enzymes with both, ATP:RF 
5'-phosphotransferase [Riboflavin kinase (RFK) (EC 2.7.1.26) (Flavokinase)] and ATP:FMN-adenylyltransferase [FMN adenylyltransferase (EC 2.7.7.2)] activities $[7,8]$ that catalyses the 5 -phosphorylation of RF to FMN and, subsequently, the adenylylation of FMN to FAD.

$$
\begin{array}{ll}
\text { RF phosphorylation } & \mathrm{RF}+\mathrm{ATP} \rightarrow \mathrm{FMN}+\mathrm{ADP} \\
\text { FMN adenylylation } & \mathrm{FMN}+\mathrm{ATP} \rightarrow \mathrm{FAD}+\mathrm{PP}_{\mathrm{i}}
\end{array}
$$

The FMN adenylylation reaction of FADS is a reversible process, while phosphorylation of RF appears to be irreversible [9]. Additionally, FADS has been shown to function with a broad variety of RF isoesters, and it is, therefore, widely used in the preparation of FMN and FAD analogues $[8,10]$. A kinetic mechanism for the steady-state reaction of the FADS from Corynebacterium ammoniagenes (CaFADS) has been proposed [9], suggesting an order in substrate binding and product release. The FMN intermediate produced by the first reaction appears to be released by the enzyme to later rebind the enzyme as a substrate for FAD production. Both enzyme activities present important differences in their substrate requirements, including concentration dependence, specificity for divalent cations and optimal $\mathrm{pH}$ or temperature $[7,11,12]$.

The C-terminal region of FADS ( $\sim 150$ residues) shares considerably sequence similarity with eukaryotic monofunctional RFKs. Likewise, the N-terminal region has been proposed to present remote similarity to nucleotydyltransferases (NTs), suggesting its involvement in the adenylylation process [13]. The structure of bifunctional FADS has only been reported for the Thermotoga maritima enzyme (TmFADS), both free and in complex with some substrates $[14,15]$. This structure shows that the enzyme is folded in two domains and it also confirms the presence of one ATP-binding site in each of the domains and a single flavin-binding site $[14,15]$. So far, neither functional studies of the TmFADS nor FADS structures from any other species have been reported.

A sequence analysis of the bifunctional FADS family and an in silico structural model for the CaFADS are here presented. Based on these data, a preliminary functional analysis of the CaFADS has been performed. Several residues are shown to be exclusively involved in either the first or second catalytic event. Additionally, the C- and Nterminal domains have been independently produced and assayed for their activities. The structural information here provided might help to envisage the rational design of selective antimicrobial drugs with the function of inhibiting FMN or FAD production and therefore, the availability in the cell of enzymes depending on these cofactors.

\section{Methods \\ Sequence searches and alignments}

The primary structure of CaFADS (sp|Q59263|RIBF_ CORAM) was obtained from the UniProtKB/SwissProt database [12]. A SIB-BLAST search of the complete CaFADS amino acid sequence was performed at http:// www.expasy.ch/tools/blast/[16] against the Eukaryota and Bacteria+Archaea subsections of the non-redundant data base UniProt Knowledgebase (Swiss-Prot + TrEMBL). Multiple sequence alignment was generated using the CLUSTAL W algorithm http://www.ebi.ac.uk/clustalw/ with default parameters [17]. Graphical representation of the multiple sequence alignment was visualised using the WebLogo server http://weblogo.berkeley.edu (Figure 1) [18]. The sequence numbering used throughout the paper corresponds to CaFADS.

\section{Prediction of a three-dimensional model for the structure of FAD synthetase from $C$. ammoniagenes}

The structural model for $C a$ FADS was constructed using homology modelling procedures based on multiple structure-base sequence alignments (including all the proteins in the PDB) as implemented in the Geno3D Web server [19]. This program uses distance geometry, simulated annealing and energy minimisation algorithms to build the protein models. The structural quality of the models was checked using the PROCHECK validation program [20]. The structures of TmFADS (1mrz, 1s4m, 1t6x, 1t6y) $[14,15]$ and those of RFKs from H. sapiens (1q9s, $1 \mathrm{p} 4 \mathrm{~m}$ ) $[21,22]$ and $S$. pombe $(1 \mathrm{n} 06,1 \mathrm{n} 08)[23]$ were suitable as templates. Six different combinations of templates were used and three models were generated by each combination.

\section{Prediction of substrate binding sites}

Predictions of putative ATP, $\mathrm{Mg}^{2+}$ and FMN binding sites of CaFADS for FMN adenylylation at the N-terminal domain were carried out by comparing our models with the structures of the nicotinamide mononucleotide adenylyltransferases (NMNAT) in complex with their substrates (Table 1). Similarly, to model ATP, $\mathrm{Mg}^{2+}$ and RF binding to the C-terminal domain of CaFADS, the structures of two RFKs in complex with their substrates (Table 1 ), as well as the different structures reported for TmFADS bound to its different substrates (Table 1), were used. O [24], Spdb-Viewer 3.7 [25] and PyMol 0.99 softwares [26] were used for complex modelling, structural analysis and figures production.

\section{Cloning and production of C. ammoniagenes wild-type and mutated FAD synthetase forms in $\mathrm{E}$. coli}

Following the Ethical Rules established at the Universidad de Zaragoza, strain DSM 20305 (ATCC 6872) from C. ammoniagenes was grown at $30^{\circ} \mathrm{C}$ in a culture medium containing $0.5 \%$ glucose, $0.5 \% \mathrm{NaCl}, 1 \%$ tryptone and 

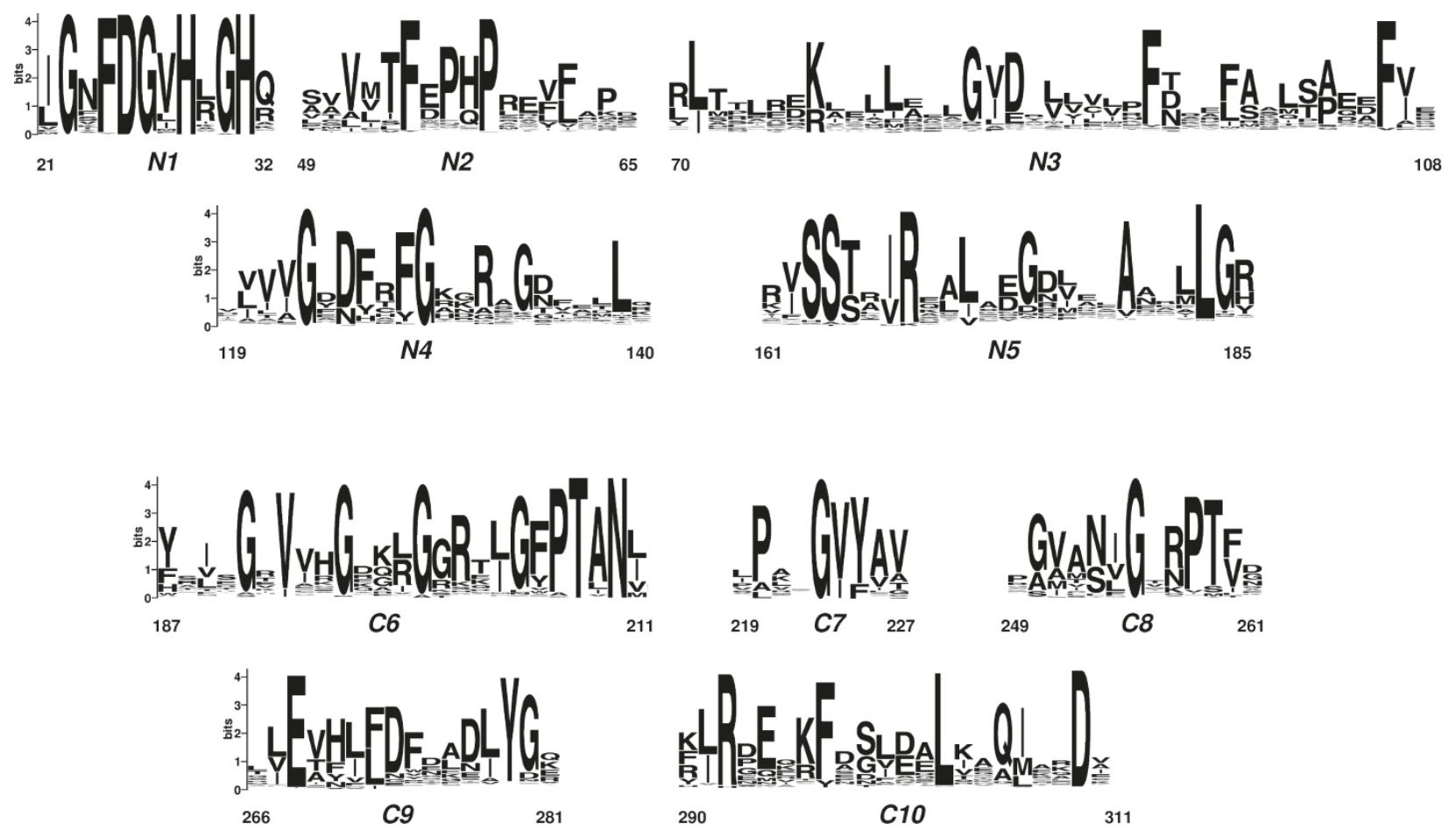

Figure I

Sequence logos including the bifunctional FADS family consensus regions and conserved residues. Numeration of CaFADS is used. The data of this logo is based on 500 sequences from the bacteria+archaea alignment of the FADS family. The sequence logo was produced using the WebLogo server http://weblogo.berkeley.edu[ [8].

$0.5 \%$ yeast extract, $\mathrm{pH} 7.2-7.4$. Genomic DNA was isolated from the cells as previously described [27]. The CaFADS encoding sequence was then amplified by the polymerase chain reaction using the forward 5'-GTAAGCCATGGATATTTGGTACGGAACAG-3' and reverse 5'-CCATCGATAGCGGATCCGGCATATAC-3' primers, synthesised on the basis of the published nucleotide sequence of the enzyme [12]. The primers incorporate the NcoI and BamHI sites respectively, and have the transcription start codon GTG replaced with ATG. The amplified 1237 bp DNA fragment was then cloned in the NcoI/BamHI sites of the pET-28a(+) expression vector. The resulting recombinant plasmid, PET28a-FADS, was used to transform E. coli BL21(DE3) competent cells. The transformants were selected on solid LB/agar medium containing $30 \mu \mathrm{g} / \mathrm{ml}$ kanamicin. The fragment was sequenced to confirm the accuracy of the amplification. Mutations were introduced into the cloned structural gene encoding the WT CaFADS by site-directed mutagenesis. The H28A, H28D, H31A, H31D, R161A, R161D, S164A, S164D, T165A, T165D, T208A, T208D, N210A, N210D, E268A and E268D FADS mutants were generated using the QuikChange mutagenesis kit (Stratagene) in combination with the adequate synthetic oligonucleotides. The gene codifying for the individual N-terminal domain (PET28a- $\Delta(183-338) F A D S)$ was obtained replacing the codon codifying for Leu183 in PET28a-FADS with a stop codon, following the methodology above mentioned for production of site-directed mutants. The C-terminal domain was individually cloned (PET28a$\Delta(1-182) \mathrm{FADS})$ using the protocol described for WT CaFADS with the forward primer 5'-CCAACTGGGCCATGGGGCGGCAC-3' and the reverse primer above used for the full length protein cloning. The forward primer used to clone the C-terminal domain incorporates the start codon ATG in a NcoI site substituting for Leu183. Mutations were ver- 
Table I: Structures used to model CaFADS and the interaction with its ligands.

\begin{tabular}{|c|c|c|c|c|c|}
\hline & PDB & SUBSTRATES & (\%) IDENTITIES & (\%) CONSERVATIVE SUBSTITUTIONS & REFERENCE \\
\hline \multicolumn{6}{|l|}{ FAD synthetases } \\
\hline T. maritima & Imrz & & 29.3 & 40.2 & [14] \\
\hline $\mathrm{N}$-terminala & & & 30.6 & 39.2 & \\
\hline C-terminalb & & & 27.6 & 41.5 & \\
\hline \multicolumn{6}{|l|}{ T. maritima complexed } \\
\hline & $\mathrm{ls} 4 \mathrm{~m}$ & RF & 29.3 & 40.2 & {$[15]$} \\
\hline & $1+6 x$ & ADP & & & \\
\hline & It6y & FMN, ADP, AMP & & & \\
\hline \multicolumn{6}{|l|}{ Nucleotydyltransferases $^{\mathrm{a}}$} \\
\hline GCT B. subtilis & I coz & CTP & 12.4 & 23.1 & [30] \\
\hline NMNAT M. jannaschii & If9a & $\mathrm{Mg}^{2+}, \mathrm{ATP}$ & 12.9 & 22.0 & [32] \\
\hline NMNAT H. sapiens & Ikku & & 10.8 & 18.3 & {$[31]$} \\
\hline NMNAT M. thermoautotropicum & lej2 & $\mathrm{NAD}^{+}, \mathrm{Na}^{+}$ & 13.0 & 22.6 & [33] \\
\hline \multirow[t]{2}{*}{ PPAT E. coli } & $1 b 6 \mathrm{t}$ & $\mathrm{dPCoA}$ & 13.0 & 26.3 & [34] \\
\hline & $\operatorname{lgn} 8$ & ATP, $\mathrm{Mn}^{2+}$ & & & {$[37]$} \\
\hline PPAT T. thermophilus & lod6 & Ppant & 11.3 & 22.0 & [35] \\
\hline \multicolumn{6}{|l|}{ Riboflavinkinases ${ }^{b}$} \\
\hline \multirow[t]{3}{*}{ H. sapiens } & $\mathrm{Ip} 4 \mathrm{~m}$ & $\mathrm{ADP}, \mathrm{Mg}^{2+}, \mathrm{FMN}$ & 25.7 & 41.4 & {$[2 \mid]$} \\
\hline & Iq9s & $\mathrm{ADP}, \mathrm{Mg}^{2+}, \mathrm{FMN}$ & & & [22] \\
\hline & Inbo & $\mathrm{ADP}, \mathrm{Mg}^{2+}$ & & & {$[21]$} \\
\hline \multirow[t]{3}{*}{ S. pombe } & $\ln 06$ & ADP & & & [23] \\
\hline & In07 & FMN, ADP & 23 & 38.2 & \\
\hline & $\ln 08$ & $\mathrm{Zn}^{2+}, \mathrm{ADP}$ & & & \\
\hline
\end{tabular}

Parameters derived from the structure-based sequence alignment of the produced CaFADS models over structural databases.

a Alignments with the segment 19-186 from CaFADS

b Alignments with the segment 187-338 from CaFADS

ified by DNA sequence analysis. WT and mutant FADS proteins were over-expressed in E. coli.

\section{Purification of recombinant FADS from $\mathrm{C}$. ammoniagenes}

E. coli cells containing the recombinant pET28a-FADS plasmid (or its mutants) were grown in LB. During the exponential E. coli growing phase, FADS was induced by overnight incubation at $37^{\circ} \mathrm{C}$ with IPTG $(1 \mathrm{mM})$. Cells were harvested by centrifugation and stored at $-20^{\circ} \mathrm{C}$. In a typical purification, around $25 \mathrm{~g}$ of cells (over-expressing recombinant FADS) were thawed, resuspended in $125 \mathrm{ml}$ of cell disruption buffer (50 mM Tris/ $\mathrm{HCl}, \mathrm{pH} 8.0,1 \mathrm{mM}$ EDTA, $12 \mathrm{mM} \beta$-mercaptoethanol, $1 \mu \mathrm{M}$ PMSF) and broken by ultrasonic treatment at $4^{\circ} \mathrm{C}$ using 16 cycles of 1 min in a DRH UP200 DR Hielsher sonicator. The cell debris was removed by centrifugation at $40000 \mathrm{~g}$ during $45 \mathrm{~min}$. The resulting yellow FADS-containing supernatant was fractioned with $45 \%$ ammonium sulphate. The mixture was again centrifuged for $45 \mathrm{~min}$ at $4000 \mathrm{~g}$ and the supernatant was loaded onto a Phenyl-Sepharose High performance (Amersham Biosciences, GE Healthcare) column equilibrated with $50 \mathrm{mM}$ Tris/ $\mathrm{HCl}, \mathrm{pH} 8.0$, $45 \%$ ammonium sulphate. The column was washed with the same buffer containing only $17 \%$ ammonium sulphate until most of the yellow colour washed out of the column. The enzyme was eluted using a $17 \rightarrow 0 \%$ ammo- nium sulphate reversed-gradient in the same buffer. Fractions of $6-8 \mathrm{ml}$ were collected while the absorbance at $280 \mathrm{~nm}$ was recorded. Fractions presenting absorbance at $280 \mathrm{~nm}$ were analysed by SDS-PAGE and, those containing the over-expressed FADS were pooled and dialysed against $50 \mathrm{mM}$ Tris/HCl, $\mathrm{pH}$ 8.0. After dialysis the protein was loaded onto a DEAE-cellulose (DE52, Whatman, England) column equilibrated with $50 \mathrm{mM}$ Tris/ $\mathrm{HCl}, \mathrm{pH}$ 8.0. The column was washed with the equilibration buffer containing $0.1 \mathrm{M} \mathrm{NaCl}$ and subsequently eluted using a $0.1 \rightarrow 0.5 \mathrm{M} \mathrm{NaCl}$ gradient. Fractions of $5 \mathrm{ml}$ were collected while recording the absorbance at $280 \mathrm{~nm}$ and, subsequently analysed by SDS-PAGE. The enzyme was considered pure when a single band of the expected size was observed in SDS-PAGE. Pure FADS fractions were pooled together, dialysed against $50 \mathrm{mM}$ Tris/ $\mathrm{HCl}, \mathrm{pH} 8.0$ and stored at $-20^{\circ} \mathrm{C}$.

\section{Measurement of riboflavin kinase and adenylyltransferase activities of FAD synthetase}

Conversion of RF into FMN and, of FMN into FAD were qualitatively assayed by addition of the different FADS variants (final enzyme concentration $\sim 1 \mu \mathrm{M}$, calculated using a theoretical $\varepsilon_{280 \mathrm{~nm}}=27.8 \mathrm{mM}^{-1} \mathrm{~cm}^{-1}$ ) to a solution (final volume, $150 \mu \mathrm{l}$ ) containing $50 \mu \mathrm{M}$ flavin (either RF or FMN), $0.2 \mathrm{mM}$ ATP and either $0.8 \mathrm{mM}$ or $10 \mathrm{mM}$ $\mathrm{MgCl}_{2}$, in $50 \mathrm{mM}$ Tris/HCl, pH 8.0. All these chemicals 
were obtained from SIGMA and were from the highest purity available. After 30 minutes of incubation at $37^{\circ} \mathrm{C}$, the reaction was stopped by boiling the preparations for 5 minutes. Transformation of RF into FMN and FAD, or FMN into FAD, was visualised by resolving the products of the reaction at room temperature and in the dark by TLC on Silica Gel SIL-G-25 $(20 \mathrm{~cm} \times 20 \mathrm{~cm}$, thickness 0.25 $\mathrm{mm}$ ) plates [28]. The moving phase was a solution of butanol:acetic acid:water (12:3:5). Flavin TLC spots were visually examined and scanned by determining their fluorescence under an ultraviolet light. The different flavins move up the plate at different rates due to differences in their partioning behaviour between the mobile liquid phase and the stationary phase. The weaker intensity of the FAD fluorescence with regard to equivalent amounts of RF or FMN relates with its flavin fluorescence quenching upon stacking of the adenine portion [29]. An additional band, running slightly faster than FMN, is observed in some experiments. The nature of this band is not known, but it is only present in the experiments containing simultaneously FMN and FAD.

\section{Results and discussion}

\section{Sequence analysis of the FAD synthetase family}

No matches for bifunctional FADS sequences were obtained when performing the SIB-BLAST search of the $\mathrm{CaFADS}$ sequence on the UniProt Knowledgebase eukaryota subsection. When searching the bacteria+archaea database subsection $(2,929,087$ sequences searched $)$, the 777 sequences displaying E-values $\leq 0.009$ were chosen for further analysis. Redundant sequences from the same species presenting sequence identity over $80 \%$ were eliminated. The remaining sequences included some proteins noticeably shorter as well as proteins lacking the typical consensus sequences found in FADSs. These sequences (less than 5\%), most of them displaying the lowest E-values of the selected sequences, were removed and separately analysed. They could be sorted out into several groups: i) proteins that present only the $\mathrm{N}$-terminal region of FADS, ii) proteins that present only the C-terminal region of FADS, iii) proteins that lack the first conserved motif in the N-terminal region, iv) proteins with a conserved $\mathrm{N}$-terminal region and a C-terminal region that shares no similarity with the bifunctional FADS family, and $\mathrm{v}$ ) proteins with a conserved C-terminal region and a $\mathrm{N}$-terminal region that shares no similarity with the bifunctional FADS family (in Rhodococcus sp. RHA1, the protein $\mathrm{N}$-terminal region is $40 \%$ identical to 3,4-dihydroxy-2-butanone-4-phosphate synthase, an enzyme involved in RF biosynthesis). Some of these divergent proteins belong to organisms that also possess a typical prokaryotic FADS sequence.

After the manual refinement, a multiple sequence alignment over the remaining 500 FADS-like sequences, dis- playing E-values $\leq 2 \mathrm{xe}^{-6}$ with $\mathrm{CaFADS}$, was carried out. Only FADSs from the genus Corynebacterium displayed sequence identities over $50 \%$ with the CaFADS (up to $60 \%$ identity in the case of the FADS from C. efficiens). However, the multiple alignment pointed out several highly conserved residues and motifs (Figure 1). Motifs 21- $\Phi-G-\Omega-F-D-G-\Phi-H-\Omega-G-H-\Omega-32$ and 162- $\Phi-S-S-[T S]-$ $\Omega$-[IV]-R- $\Omega-\Omega-\Phi-\Omega-\Psi-G-174 \quad(\Phi, \quad \Psi$ and $\Omega$, denoting hydrophobic, polar and any residue, respectively) (N1 and N5 in Figure 1) corresponded to two consensus sequences in the FADS family that are also conserved in NTs remotely related to FADS (see below). Two additional consensus sequences in between the above mentioned motifs were also observed. They included residues $50-\Omega$ -

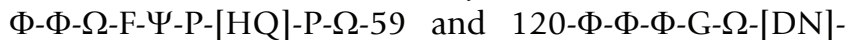
$[\mathrm{FYH}]-\Omega-[\mathrm{FY}]-\mathrm{G}-\Omega-129$ (N2 and $\mathrm{N} 4$ in Figure 1 ) and, apparently, only the motif $123-\mathrm{G}-\Omega-\Psi-125$ presents an equivalent in NT sequences (see below). Multiple sequence alignment also showed a number of residues highly conserved, or conservatively substituted, between residues 1 and 186: I71, R77, G85, I86, D87, F94, F98, Y106, V107, A179, L183 and G184 (CaFADS numbering). These residues are mainly included in region N3 and at the end of N5 in Figure 1 and, can be considered as forming additional consensus sequences. Noticeably, only R77 and A179 are conserved in NTs.

The C-terminal region of the bifunctional FADS sequences (residues 187-338) also included several consensus sequences, $191-G-\Omega-V-\Phi-\Psi-G-\Omega-\Omega-\Omega-G-\Omega-\Psi-\Phi-203,205-$ G-[FY]-P-T-[ALIV]-N-210, 223-P- $\Omega-\Omega-G-[V I]-[Y F]-225$,

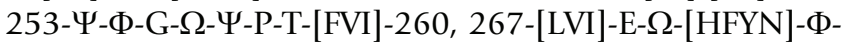
[FL]-[DN]-[FWY]- $-\Phi-[D N E]-[L I A V]-Y-[G D N]-280$ and 291-[LI]-R- $\Omega$-[EQMN]- $\Omega$-[KRT]-[FY]-296, and the highly conserved residues L303 and D310 (see C6-C10 in Figure 1 ), being all of them also conserved in the sequences of RFKs (see below).

\section{Structural model of the FAD synthetase from C. ammoniagenes}

A 3D structural model for the CaFADS has been produced based on the structure of the TmFADS (Figure 2) [14,15]. The low sequence similarity of the N-terminal domain of FADSs with NTs prevented these enzymes to be used as templates (Table 1, Figure 3A) [13], but the sequence similarity found at the C-terminal domain of the FADS family with RFKs from $H$. sapiens $[21,22]$ and $S$. pombe (Table 1 , Figure 3B) [23] allowed them to be used as templates. These RFKs were used for the construction of the model in those loops were the X-ray structure of TmFADS did not show experimental information [14,15]. Models for the $\mathrm{CaFADS}$ C-terminal domain generated using as templates only the structures of RFKs were consistent with the C-terminal structure reported for TmFADS, indicating that RFKs are a good choice as templates for this FADS family 
domain. Stereochemistry and energy analysis of the produced models suggested that the model constructed using the FADS and RFK templates reported in Table 2 (Figure $2 \mathrm{~A}$ ) was adequate to describe the $3 \mathrm{D}$ structure of $\mathrm{CaFADS}$ until structures are provided by experimental methods.

According to the model, the protein folds in two domains. The N-terminal domain (residues 18-186) lacks the first 18 residues of the sequence (not present in the TmFADS template), while the C-terminal domain (residues 187318 ) lacks the last 20 residues (templates were shorter in sequence or presented very low sequence similarity in those residues) (Figures 2 and 3). The main structural arrangements predicted for the CaFADS are present in the structure reported for the TmFADS [14,15], but our model also showed the disposition of some loops and a 310helix at the C-terminal domain that are missed in the TmFADS structure. Additionally, the C-terminal domain of CaFADS showed an insertion around residue 230 with regard to TmFADS and many members of the FADS family.

\section{The kinase domain: the C-terminal domain of $\mathrm{C}$. ammoniagenes FAD synthetase}

The C-terminal domain of the CaFADS model (residues $187-318)$ folds in a central six-stranded antiparallel $\beta$ barrel stabilised between the single final $\alpha$-helix $(\alpha 7)$, the two small $\alpha 5$ and $\alpha 6$ of the N-terminal domain and several loops (Figure 2A). A short $3{ }_{10}$-helix is also predicted in our model between residues G201-L204. This C-terminal domain showed a high sequence identity in the 3D space with the structures of the templates (Figures $3 \mathrm{~B}$ and $4 \mathrm{~A}$, Table 1) and, there is no doubt that it must contribute to ATP, $\mathrm{Mg}^{2+}$ and RF binding to produce FMN. Comparison of this model with the reported TmFADS and RFKs structures in complex with their substrates will provide information about $\mathrm{CaFADS}$ residues involved in substrate binding and catalysis (Figures $3 \mathrm{~B}$ and $4 \mathrm{~B}$ ). Therefore, it is expected that the ADP- $\mathrm{Mg}^{2+}$, or ATP- $\mathrm{Mg}^{2+}$, will be nested at one edge of the $\beta$-barrel, between two loops (Figure $4 \mathrm{~B})$. The first loop (R195-N210) contains the short $3_{10^{-}}$ helix and the 207-РТФN-210 consensus sequence conserved in RFKs (Figures 1 and 3B) and, it is proposed to stabilise the P groups (Figure 4B). The structures of RFKs in complex with ADP-Mg2+ also suggest that T208 and N210 might contribute to metal binding $[22,23]$. The second loop (E268-Y279) starts with the E268 residue, invariant in FADS and RFK families and proposed to link the terminal $\mathrm{OH}$ group of the RF ribityl chain, acting as a catalytic base [21-23]. This loop also includes the D277 and Y279 conserved positions, proposed to stabilise the ATP adenine ring, while V193, K202 and D277 might contribute to stabilise the ribose (Figures 1 and $4 \mathrm{~B}$ ) by similarity with RFKs $[21,23]$. Comparison of models with TmFADS and RFKs complexed with RF or FMN suggests that the isoalloxazine ring must be bound in a pocket formed by the outer surface of the $\beta$-barrel $(\beta 8-\beta 10)$ and the long terminal $\alpha 7$ helix. The flavin ring might result stabilised by the hydrophobic environment provided by the consensus region 223-G-V-Y-225 and the conserved F206, F270, F297, L303 and M307 residues, while its hydrophilic portion may be pointing towards the opening of the pocket and stabilised by E295 or K296 [15,21,22]. Other residues apparently not directly involved in flavin binding but contributing to the formation of the bottom and top of the pocket include the consensus region 254-G- $\Omega-\Psi-P-T-$ [FVI]-260 and the highly conserved residues R292 and D310, respectively. These two later residues apparently form a salt bridge that contributes to fix the terminal $\alpha$ helix close to the top of the flavin ring cavity (Figure 4B). The ribityl chain would extend towards $\beta 7$, interacting as above mentioned with E268 (Figure 4B).

The models for $C a$ FADS suggest flexibility in the loops connecting $\beta 6-\beta 7$ and $\beta 9-\beta 10$ (Figure $2 \mathrm{~B}$ ). Both of these regions are not solved in the structures reported for the TmFADS $[14,15]$. Flexibility is also observed in regions involved in substrate binding (Figure 4B), in agreement with conformational changes observed in RFKs upon substrate interaction [22]. These conformational changes provide additional interactions, for both the ribityl tail and the isoalloxazine ring and, help to anchor the reactive substrates and the catalytic residues in the most adequate disposition for catalysis [21-23]. This analysis also suggests that despite RFKs and FADSs surely share a similar mechanism in the RF phosphorylation reaction, variations in the structural details for substrate binding and catalysis can be expected.

Table 2: R.m.s.d. (Å) between the three predicted models for CaFADS and the structures used for their production.

\begin{tabular}{|c|c|c|c|}
\hline Structure & Model I & Model 2 & Model 3 \\
\hline Model I & 0.00 & 1.34 & 1.49 \\
\hline Model 2 & 1.34 & 0.00 & 1.22 \\
\hline Model 3 & 1.49 & 1.22 & 0.00 \\
\hline T. maritima FADS (I mrz) & 1.53 & 1.49 & 1.44 \\
\hline T. maritima FADS, RF (Is4m) & 1.60 & 1.56 & 1.39 \\
\hline H. sapiens RFK, ADP, $\mathrm{Mg}^{2+}$, FMN (Ip4m) & 1.74 & 1.54 & 1.25 \\
\hline S. pombe RFK, ADP, $\mathrm{Zn}^{2+}(\operatorname{In} 08)$ & 1.82 & 1.67 & 1.60 \\
\hline
\end{tabular}



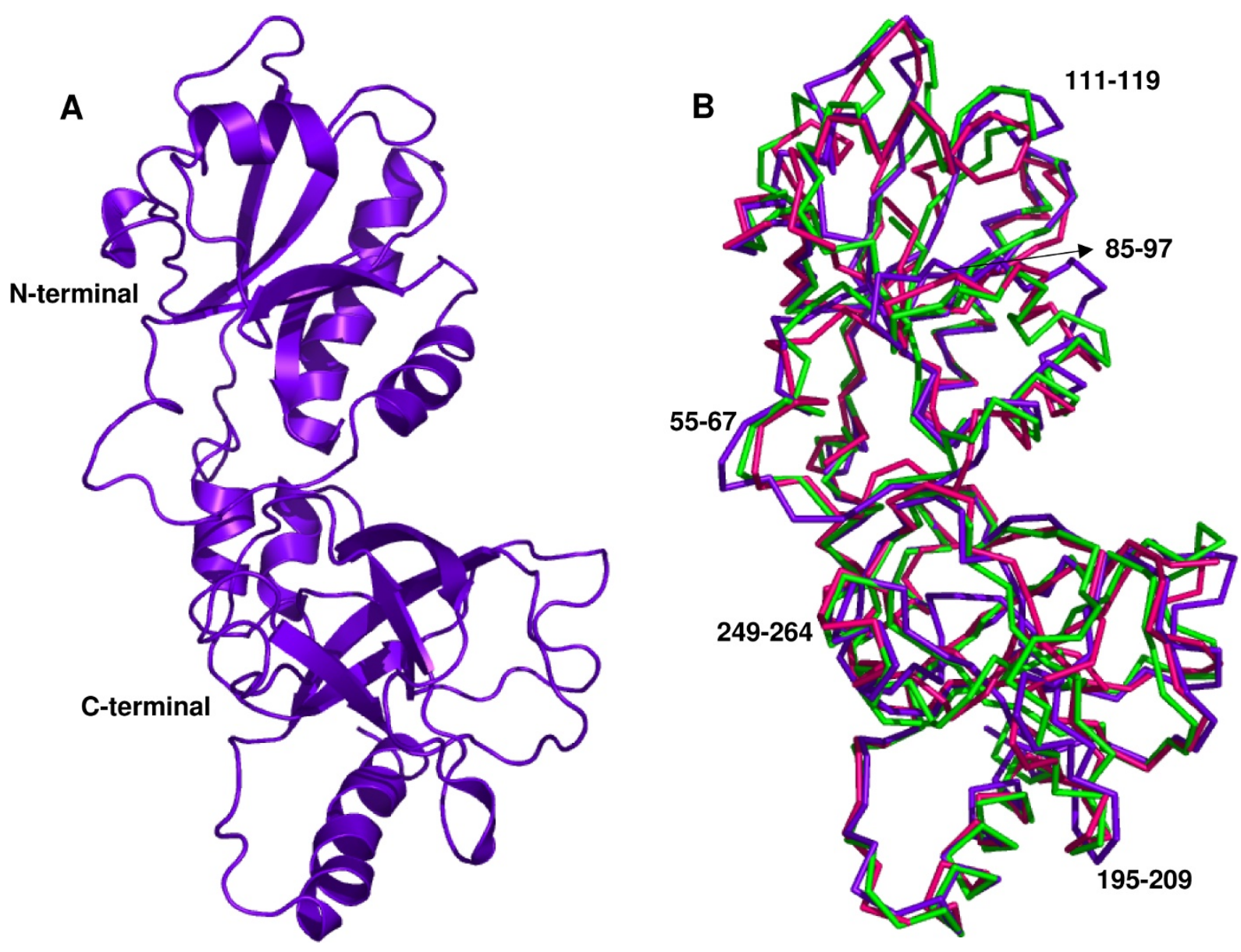

Figure 2

Predicted structural model for CaFADS. (A) Ribbon representations of the model selected to represent CaFADS (Table 2). (B) Superposition of the three models generated in this prediction. Residues involved in putatively high flexible regions are indicated.

The adenylyltransferase domain: the $\mathbf{N}$-terminal domain of C. ammoniagenes FAD synthetase

The CaFADS N-terminal domain consists of a classical $\alpha /$ $\beta$ dinucleotide binding domain. Its core is formed by a twisted five-stranded parallel $\beta$-sheet flanked by two long and two short $\alpha$-helices and the corresponding connecting loops (Figure 5). The end of this domain forms a small subdomain made up of two small $\alpha$-helices ( $\alpha 5$ and $\alpha 6$ ), which are in contact with the C-terminal domain. The CaFADS model was compared with the structures reported for several enzymes of the NT $\alpha / \beta$ phosphodiesterase superfamily (Table 1, Figures $3 \mathrm{~A}$ and $5 \mathrm{~A}$ ), either free or in complex with their substrates [13-15,30-35]. Members of this family catalyse the transfer of a nucleotide monophosphate moiety, from a nucleotide triphosphate (NTP), onto different substrates. Our CaFADS Nterminal model presents an overall folding conserved in
NTs (Figure 5A) that allowed improvement of the sequence alignments and production of putative models for the interaction between this CaFADS domain and its substrates (Figures 3A, 5 and 6). The consensus regions 19-32 and 162-174, highly conserved in the FADS and NT families (Figures 1 and $3 \mathrm{~A}$ ), are proposed to be involved in NTP stabilisation in the active site. In our model these motifs include the $\alpha 1$ helix (H28-V45), placed between $\beta 1$ and $\beta 5$, the E158-S164 loop and some portions of the two terminal antiparallel $\alpha$-helices, ( $\alpha 5$ (T165-S172) and $\alpha 6$ (D175-L183)) (Figure 5). Residues of these consensus regions show a high conservation in primary and 3D structures when comparing the FADS and NT families. Thus, in CaFADS: i) main-chain atoms of G22, F24 and D25 might H-bond the $\alpha$-P of ATP, ii) H28, G30 and H31 (N-terminal of helix $\alpha 1$ ) might contribute to stabilise the P groups and adenine moiety of ATP, iii) 


\section{$\underline{\text { A. N-terminal alignment }}$}

$\begin{array}{ll} & \\ \text { CaFADS } & 19 \\ 1 \mathrm{t} 6 \mathrm{y} & 1 \\ 1 \mathrm{coz} & 1 \\ 1 \mathrm{kku} & 7 \\ 1 \mathrm{ej} 2 & 4 \\ 1 \mathrm{f} 9 \mathrm{a} & 1 \\ 1 \mathrm{~b} 6 \mathrm{t} / 1 \mathrm{gn} 8 & 3 \\ 1 \mathrm{od} 6 & 1 \\ \text { Consensus } & \text { region }\end{array}$

$$
\beta \beta \beta \quad \alpha \alpha \alpha \alpha \alpha \alpha \alpha \alpha \alpha \alpha \quad \alpha \alpha \alpha \alpha \quad \beta \beta \beta \beta \beta \quad \alpha \alpha \alpha \alpha \alpha \alpha \alpha
$$

\section{$\bullet \bullet \bullet \bullet \triangle \Delta \quad \bullet$}

V-TIGV FDGVHRGHQK LINATVE--- ----KAREVG AK-AIMVTFD -PHPVSVFLP RRAPLGITTL -AERFA-LAE ---------S MVV-SIGV FDGVHIGHOK VLR-TMKE-- ----IAFFRK DD-SLIYTIS Y--PPEYFLP DFPGLIMTV- -ESRVE-MLS --------R MKKVI-TYGT FDLLHWGHIK LLERAKQ--- ----LGD--- -Y-LVVAIST DEFNL--QKQ KKAYHS---- YEHRKL-ILE ---------T TEVVLLACGS FNPITNMHLR LFELAKDYMN GTGRYTV--- -VKGIISPVG DAY------ KKKGLIPA- Y-HRVIMAEL ATKNSKWVEV MRGL-LVGR MQPFHRGHLQ VIKSILE--- ----EVDE-- ---LIICIGS AQLS----HS IRDPFT---- AGERVM-MLT --------K LRGF-IIGR FQPFHKGHLE VIKKIAE--- ----EVDE-- ---IIIGIGS AQKS----HT LENPFT---- AGERIL-MIT --------Q KRAI-YPGT FDPITNGHID IVTRATQ--- ----MFD--- -H-VILAIAA SPS------ KKPMFTL--- -EERVA-LAQ --------Q MHVV-YPGS FDPLTNGHLD VIQRASR--- ----LFE--- -K-VTVAVL- -EN------ - -QYLFSAEE ---RLAIIR- -----EATA $G \Omega \quad F D \Omega \Phi H \Omega G H \Omega \Omega$

\begin{tabular}{|c|c|c|c|c|c|c|c|c|}
\hline & $\beta \beta \beta \beta \beta \beta$ & ${ }_{* \nabla}^{\alpha}{ }^{\alpha} \alpha$ & $\begin{array}{ll}\alpha \alpha & \alpha \alpha \\
\Delta & \nabla \Delta^{2}\end{array}$ & $\alpha \alpha$ & $\begin{array}{c}\beta \beta \beta \beta \beta \\
* * \bullet\end{array}$ & * & $\alpha$ & \\
\hline FG-------- & IDGVLVIDFT & & $\begin{array}{l}\text { PEK----YVE } \\
\text { VFR----YT S }\end{array}$ & FLLED--TLH & $\begin{array}{l}\text { AS-HVVVGA- } \\
\text { VS-AVVVGR- }\end{array}$ & $\begin{array}{l}-------\mathrm{N}- \\
-\end{array}$ & FTFG---ENA & - \\
\hline $\begin{array}{l}\text { YA--------- } \\
\text { IR------YYV }\end{array}$ & $\begin{array}{l}\text { RTVVLDF-FR } \\
\text { DE-VIPEK-- }\end{array}$ & $\begin{array}{l}\text { IKDL-TPEGF } \\
---------\end{array}$ & $\begin{array}{l}\text { VER----YLS } \\
\text {-NWEQKKQ-D }\end{array}$ & $\begin{array}{l}\text { G--------- } \\
\text { I------IDH }\end{array}$ & $\begin{array}{l}\text { VS-AVVVGR- } \\
\text { NIDVFVMG-- }\end{array}$ & $\begin{array}{l}-------D- \\
-----D--D-\end{array}$ & $\begin{array}{l}\text { FRFG---KNA } \\
------ \text { WEG }\end{array}$ & $\begin{array}{l}---------- \\
\text { K-FDFL---- }\end{array}$ \\
\hline DT------WE & SL-QKEWK-- & ---------- & -ETLKVLR-H & HQEKLEASDV & PKVKLLCGA- & ----DLLES & F-AVPNLWKS & EDITQI-_-_- \\
\hline ALSENGIPAS & RYYIIPVQ-- & --DIECNAL- & WVGH IKML-- & $-------T P$ & PFDRVYSG-- & $------N-$ & PLVQRLFSED & \\
\hline KD---YDL & TYYPIPIK-- & --DIEFN--- & -SIWVS-YVE & S------LTP & PFDIVYSG-- & $-----\mathrm{N}-$ & ---- & --PLVR \\
\hline$---\mathrm{GN}$ & VEVVG & --- & ----ANFARN & $-\mathrm{HA}-$ & -T-VLIRGLR & FEY-E- & & QLAH-MNRHL \\
\hline$---\mathrm{AN}$ & $\mathrm{VE}-\mathrm{AA}$ & -- & VD-F & V-------RRV & $\begin{array}{c}\text { GAQAIVKGLR } \\
G \Omega\end{array}$ & $\begin{array}{c}\text { AVSDYEY-EL } \\
\Psi\end{array}$ & QMAHLN---- & \\
\hline$\alpha \alpha$ & $\beta \beta \beta$ & $\begin{array}{l}\beta \beta \beta \\
\Delta\end{array}$ & & - * & • $\quad \nabla^{\alpha}$ & $\alpha \alpha \alpha \alpha \alpha \quad \alpha$ & $\alpha \alpha \alpha \alpha$ & $\alpha$ \\
\hline RQI & CQSR-LTV & ---- IDLLD & & & GV-R--ISST & TVREI & DVI & \\
\hline $\begin{array}{l}\text { SGNASFLR-- } \\
--------\end{array}$ & $\begin{array}{l}\text {-KKG-VEVYE } \\
--K D Q C E V-V\end{array}$ & $\begin{array}{l}-----I--E D \\
-----Y L P-R\end{array}$ & 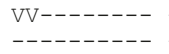 & $\begin{array}{l}-------V Q- \\
------\mathrm{TE}-\end{array}$ & $\begin{array}{l}\text { GK-R--VSSS } \\
--- \text {-G--ISTT }\end{array}$ & $\begin{array}{l}\text { LIRNLV-QEG } \\
\text { KIKEEI---- }\end{array}$ & $\begin{array}{l}\text { RVEEIPAYLG } \\
-\end{array}$ & RY-------- \\
\hline -------VA- & --NYGLI-CV & TRAGNDAQKF & IYESDVLWKH & RSNIHVVNEW & --FANDISST & ALRRGQ & DLV & - \\
\hline --------- & & GYEVTAPPLF & & ----- & -- YRDRYSGT & EVRRRMLDDG & PESV & VEVID \\
\hline FEERGY-- & & --EVKRPEMF & - & -------- & N--RKEYSGT & EIRRRMLNGE & KWEHLVPKAV & VDVI \\
\hline P------EL & ES-------- & ----- VFLMP & --- & ------SKEW & -SF---ISSS & LVKEVA-HQG & ---DVTHELP & ENVH \\
\hline$---\mathrm{RQL}$ & YPGLETL--- & FILAATRYSF & ------ & ---------- & $\begin{array}{r}-----V S S T \\
\Phi S S T\end{array}$ & $\begin{array}{l}\text { MVKE IARYGG } \\
\Omega V R \Psi\end{array}$ & DVSKLVPPAT & LRALKAKLGQ \\
\hline
\end{tabular}

\begin{tabular}{|c|c|c|c|c|c|c|c|c|c|c|}
\hline & & & $\beta \beta \beta \beta \beta \beta$ & $\underset{* \nabla}{\alpha} \alpha$ & $\alpha \alpha \quad \alpha \alpha$ & $\alpha \alpha$ & $\begin{array}{c}\beta \beta \beta \beta \beta \\
* * \bullet \bullet\end{array}$ & * & $\alpha$ & \\
\hline CaFADS & 84 & FG-------- & IDGVLVIDFT & RELSGT---S & PEK----YVE & FLLED--TLH & AS-HVVVGA- & $-\mathrm{N}-$ & FTFG---ENA & \\
\hline $1 \mathrm{t} 6 \mathrm{y}$ & 66 & YA-------- & RTVVLDF-FR & IKDL-TPEGF & VER----YLS & 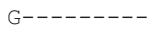 & VS-AVVVGR- & $-------D-$ & FRFG---KNA & \\
\hline $1 \mathrm{coz}$ & 62 & IR------YV & DE-VIPEK-- & --- & -NWEQKKQ-D & I-----IDH & NIDVFVMG-- & $-----D--D-$ & ------ WEG & $\mathrm{K}-\mathrm{FDFL}-$ \\
\hline $1 \mathrm{kku}$ & 83 & DT------WE & SL-QKEWK-- & ---------- & -ETLKVLR-H & HQEKLEASDV & PKVKLLCGA- & ---- DLLES & F-AVPNLWKS & EDITQI-- \\
\hline $1 e j 2$ & 62 & ALSENGIPAS & RYYIIPVQ-- & --DIECNAL- & WVGHIKML-- & $-------\mathrm{TP}$ & PFDRVYSG-- & $-------\mathrm{N}^{-}$ & PLVQRLFSED & \\
\hline $9 a$ & 59 & SLKD---YDL & TYYPIPIK-- & --DIEFN--- & -SIWVS-YVE & S-----LTP & PFDIVYSG-- & $-----\mathrm{N}_{-}$ & - & $-----P L$ \\
\hline $6 t / 1 \mathrm{gn} 8$ & 58 & ATAHL---GN & VEVVGFSDLM & ---------- & ----ANFARN & $\mathrm{Q}------\mathrm{HA}-$ & -T-VLIRGLR & AVADFEY-E- & $--------M$ & QLAH-MNRHL \\
\hline$d 6$ & 61 & $\mathrm{HL}------\mathrm{AN}$ & VE-AATFS-- & --- & ---GLLVD-F & $\mathrm{V}------\mathrm{RRV}$ & GAQAIVKGLR & AVSDYEY-EL & QMAHLN---- & \\
\hline \multirow{2}{*}{ Consensus } & regior & & & & & & $\mathrm{G} \Omega$ & $\Psi$ & & \\
\hline & & $\alpha \alpha$ & $\beta \beta \beta$ & $\beta \beta \beta$ & & - * & • $\quad \nabla^{\alpha}$ & $\alpha \alpha \alpha \alpha \alpha \quad \alpha$ & $\alpha \alpha \alpha \alpha \alpha \alpha \alpha \alpha$ & $\alpha$ \\
\hline $\mathrm{FA}$ & 133 & AGT & CQSR-LTVDV & ---- IDLLD & & -DE- & GV-R--ISST & TVREFL-SEG & IWALG & $\mathrm{RH}--$ \\
\hline $6 y$ & 109 & SGNASFLR-- & -KKG-VEVYE & $-----I--E D$ & VV-------- & $-------V_{Q}-$ & GK-R--VSSS & LIRNLV-QEG & RVEEIPAYLG & RY--- \\
\hline$=0 z$ & 103 & --- & --KDQCEV-V & ---- YLP-R & ---------- & $------\mathrm{TE}-$ & $---G--I S T T$ & KIKEEI---- & -- & ----1 \\
\hline $\mathrm{ku}$ & 141 & ------ VA- & --NYGLI-CV & TRAGNDAQKF & IYESDVLWKH & RSNIHVVNEW & --FANDISST & KIRRALRRGQ & PDLV & ----- \\
\hline$j 2$ & 116 & ---------- & --------- & GYEVTAPPLF & -------- & --------- & --YRDRYSGT & EVRRRMLDDG & DWRSLLPESV & VEVIDEIN \\
\hline $9 a$ & 104 & VLFEERGY-- & - - - - - - - & --EVKRPEMF & ---------- & ----------- & N--RKEYSGT & EIRRRMLNGE & KWEHLVPKAV & VDVIKEIKGV \\
\hline $6 \mathrm{t} / 1 \mathrm{gn} 8$ & 110 & MP------EL & ES-------- & ---- VFLMP & -- & ----- SKEW & -SF---ISSS & LVKEVA-HQG & -- DVTHELP & ENVHQALMAK \\
\hline & 107 & $------R Q L$ & YPGLETL--- & FILAATRYSF & --------- & --------- & ----- VSST & MVKEIARYGG & DVSKLVPPAT & LRALKAKLGQ \\
\hline & & & & & & & & & & \\
\hline
\end{tabular}
Consensus region

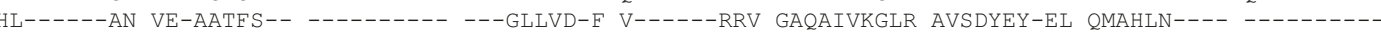

\section{B. C-terminal alignment}

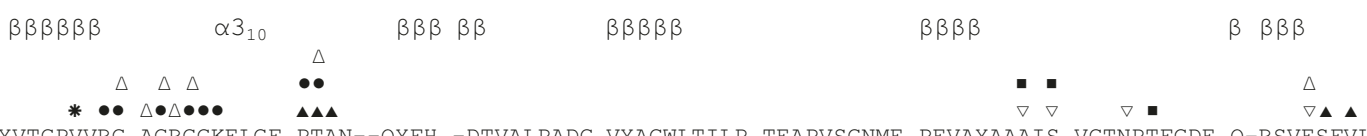

CaFADS 187 FYVTGPVVRG AGRGGKELGF PTAN--QYFH -DTVALPADG VYAGWLTILP TEAPVSGNME PEVAYAAAIS VGTNPTFGDE Q-RSVESFVI $1 \mathrm{t} 6 \mathrm{y} / 1 \mathrm{t} 6 \mathrm{x} / 1 \mathrm{~s} 4 \mathrm{~m} 160$ FEIEGIVHKD REFG-RKLGF PTANI-D-RG NEKLVDLKRG VYLVRV-HLP DGKKKFG--- -------VMN VGFRPTVGDA RNVKYEVYII $1 \mathrm{p} 4 \mathrm{~m} / 1 \mathrm{~g} 9 \mathrm{~s} / 1 \mathrm{nb0} 13$ YFCRGQVVRG FGRGSKOLGI PTANFPEOVV DNLPADISTG IYYGWASVGS GD------- ---VHKMVVS IGWNPYYKNT K-KSMETHIM 1n06/1n07/1n08 28 ----GKVVHG FGRGSKELGI PTANISEDAI QELLRYRDSG VYFGYAMVQK R--.--..-- ---VFPMVMS VGWNPYYKNK L-RSAEVHLI

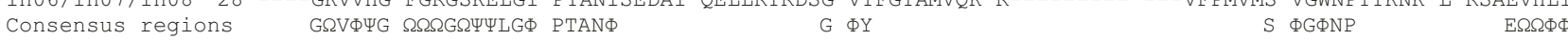

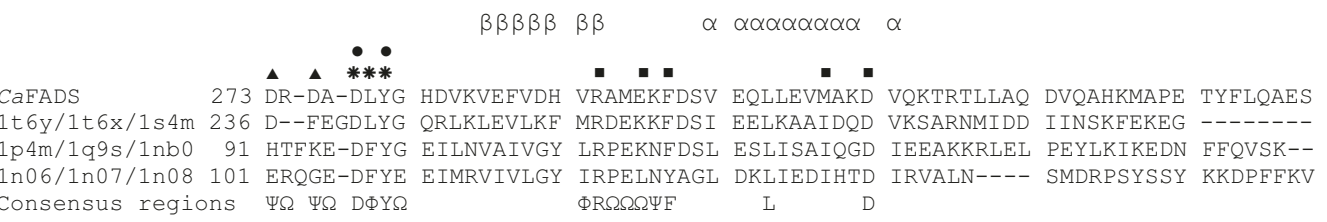

Figure 3 (see legend on next page) 
Figure 3 (see previous page)

Alignments. (A) Structural alignment of the N-terminal domain of the CaFADS model (residues 19-186) with the corresponding regions of TmFADS and several NTs. Residues proposed to be involved in particular substrate binding are marked as; for phosphate groups, * ribose or $\boldsymbol{\Delta}$ adenine portions of ATP. $\nabla$ denotes regions where substrates specific for each enzyme have been reported to interact. (B) Structural alignment of the C-terminal domain (residues I87-338) of the C. ammoniagenes FADS model with those of TmFADS and RFKs from S. pombe and $H$. sapiens. Residues proposed to be involved in particular substrate binding are marked as; for phosphate groups, * ribose or $\boldsymbol{\Delta}$ adenine portions of ATP and, $\triangle$ for the phosphate, $\nabla$ ribityl or $\mathbf{D}$ isoalloxazine ring portions of RF. Each template is referred to its pdb code according to Table I. Residues involved in substrate binding in each particular structure are shown in bold case. Consensus sequences are shown below alignment, $\Phi, \Psi$ and $\Omega$ denoting hydrophobic, polar and any residue, respectively. Secondary structure prediction of CaFADS, obtained using the JOY server [36] and visual inspection, is shown above each alignment, with $\beta$ signifying $\beta$-strand residues and $\alpha$ signifying $\alpha$-helix residues.

the ATP P groups might interact with L34, T38, I162, G174 and the 104-106 and 153-157 regions, iv) the consensus region 162-165 (including the N-terminals of $\alpha 5$ ) must be involved in stabilisation of the adenine, $\beta$-P and $\gamma$-P of ATP and, v) the 123-125 consensus motif accommodates the ribose portion of the nucleotide (Figures $3 \mathrm{~A}$ and 5), as proposed for the equivalent residues in TmFADS, glycerol-3 phosphate cytidylyltransferase (GCT), phosphopantetheine adenylyltransferase (PPAT) and NMNAT (Figure 3A, 5A) [31,32,34]. The loop formed by residues 156-160 (in red in Figure 5B), although conserved in sequence and structure, shows different orientations and lengths in the different NTs, which probably determines the enzyme specificity for the NTP (Figures 3A and 5A) [30]. The CaFADS model also showed several positively charged side-chains highly conserved in the FADS family and structurally situated in positions also occupied by positively charged side-chains in NT structures. These include R161 and R168 (R161 provided by R91 and R22 in PPAT from E. coli or T. thermophilus and, R168 by K46 in B. subtilis GCT or R47 in M. thermoautotrophicum NMNAT). Their positions suggest that these side-chains might contribute to stabilise the adenine and $P$ groups of ATP when bound to the active site. It is also worth noting that an important number positions occupied by conserved glycines in the FADS family are also conserved in sequence and structure in NTs and, in many cases, their main-chain atoms directly provide $\mathrm{H}$-bonding to the nucleotide (Figures 1 and $3 \mathrm{~A}$ ).

Flexible regions can be predicted by comparison of the CaFADS models (Figure 2B, Table 2). These, including 55-67, 85-97, 111-119 and 127-135, correspond to low similarity regions between NT and FADS families (Figures 2B, 3A and 5A). Structures of NMNAT in complex with $\mathrm{NAD}^{+}$[33], and PPAT in complex with dPCoA [34] or Ppant [35], indicate that the binding of the adenylylation substrate, $\mathrm{NMN}^{+}$or Ppant moieties, is allocated in the pocket formed by the above mentioned regions. Superposition of these complexes on our CaFADS model, followed by fitting and sculpting the FMN molecule along the nicotinamide nucleotide moiety of $\mathrm{NAD}^{+}$in the NMNAT structure, allowed production of putative models for the interaction of the FADS N-terminal domain with its FMN substrate (Figure 6A). In this model, the FMN isoalloxazine ring would be placed in a cavity surrounded by the above mentioned flexible regions. The entrance of this cavity would be flanked by the F54, F62 and D94 hydrophobic side-chains, the consensus region 54-FPHP-58 would fix the position of the ribityl of FMN, while the flavin ring environment would be contributed by hydrophobic interactions with V59, L98, Y106 and F128 (Figure 6A). All these positions are highly conserved in the FADS family, but do not have equivalence in NTs (Figures 1 and $3 \mathrm{~A})$. The model suggests that the hydrophobic isoalloxazine portion of the flavin ring might be embedded inside the cavity, while the hydrophilic edge would be partially exposed to the solvent. The size of the cavity makes it difficult to predict the precise location of the isoalloxazine ring. Various possibilities are allowed (Figure 6A) and, final disposition of loops and side-chains would depend on the flavin binding event and conformational changes not predicted in our model. This flavin binding mode could represent a novel flavin binding motif so far not described. Additionally, while sequence motifs N1, $\mathrm{N} 4$ and N5 are also conserved in the sequences of eukaryotic FMN adenylyltransferases, residues and regions proposed for flavin binding in the FADS family do not present homologous in the sequences of the monofunctional enzymes (data not shown).

This is the first structural model where a putative binding site for the flavin is suggested at the adenylylation site of FADS, indicating the presence of two independent flavin binding sites in the FADS family. An early hypothetical model for the active site of FADS proposed a single flavin binding site for both activities and two independent ATP binding sites [9]. No structural information of this family of enzymes was reported at that time, but that model accounted for the kinetic mechanism experimentally derived [9]. The 3D structures and models today available for FADS indicate that the phosphorylation and adeny- 
A

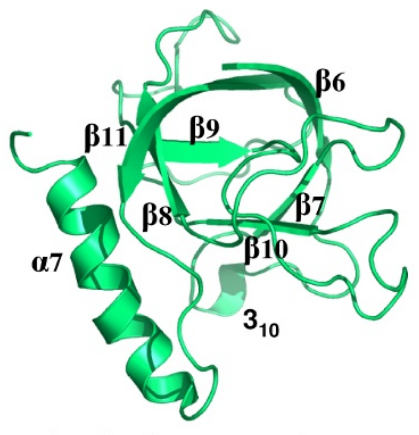

FADS C. ammoniagenes

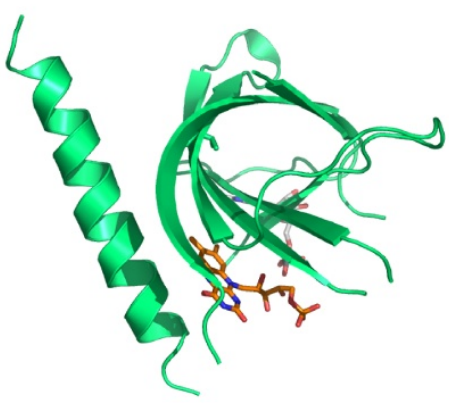

1t6y (ADP, FMN)
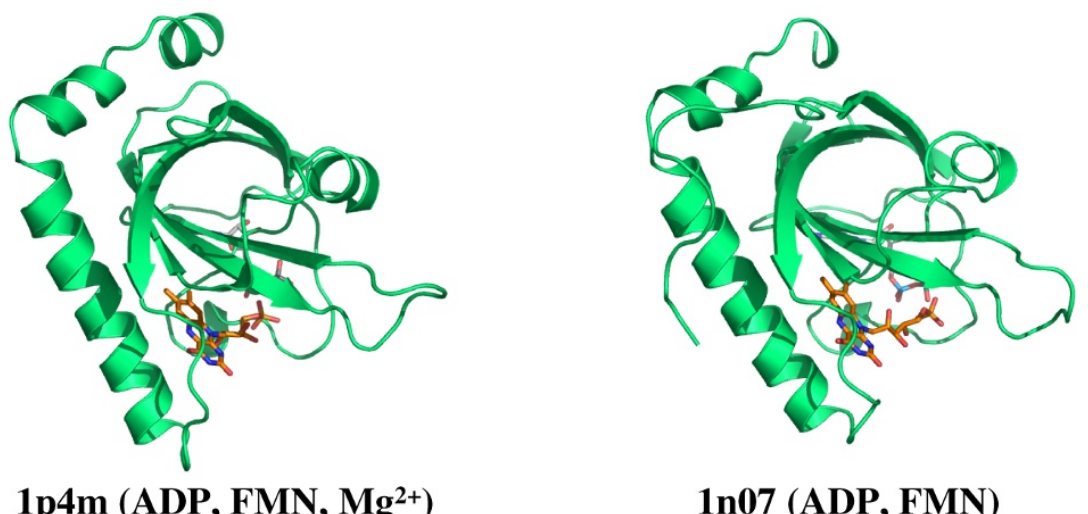

1n07 (ADP, FMN)

B

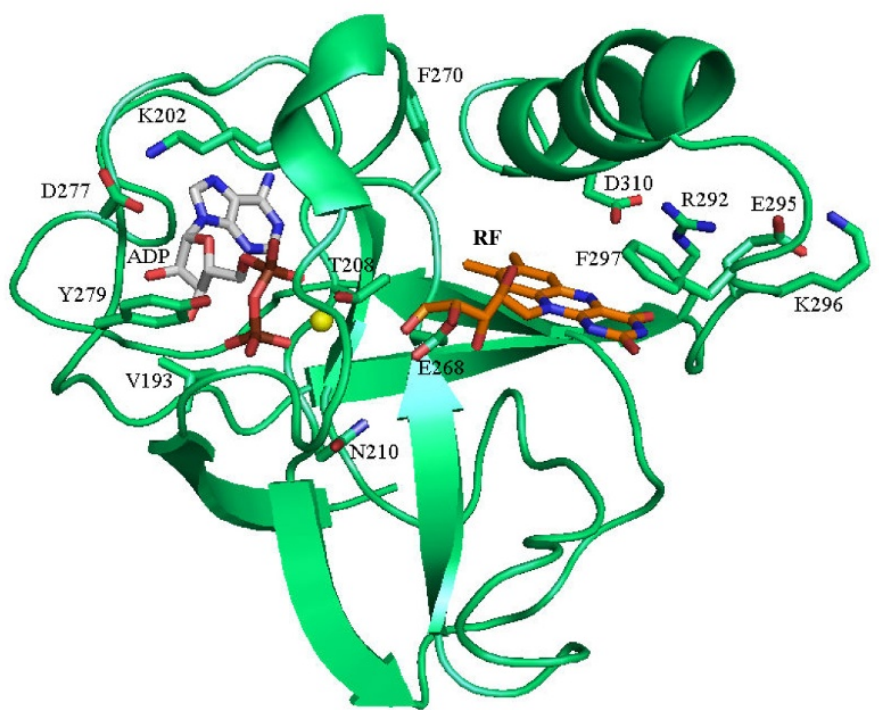

\section{Figure 4}

C-terminal domain structure ofCaFADS. (A) Comparison of the structural elements with those found in RFKs. (B) Model for the interaction of ADP-Mg2+ and RF with the C-terminal domain. Residues putatively involved at the RFK active site, nucleotides and flavins are shown in CPK sticks. Carbon atoms are shown in green, white and orange for protein side-chains, nucleotides and RF, respectively. $\mathrm{Mg}^{2+}$ ions are shown as yellow balls. 


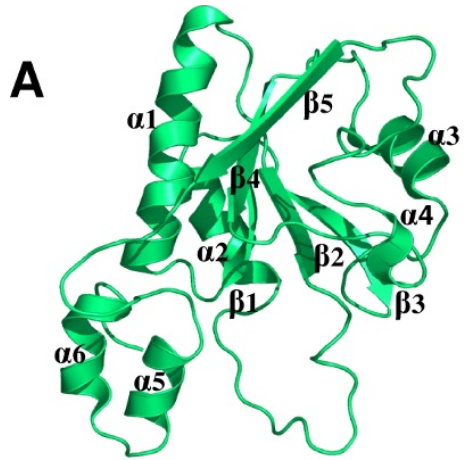

FADS C. ammoniagenes

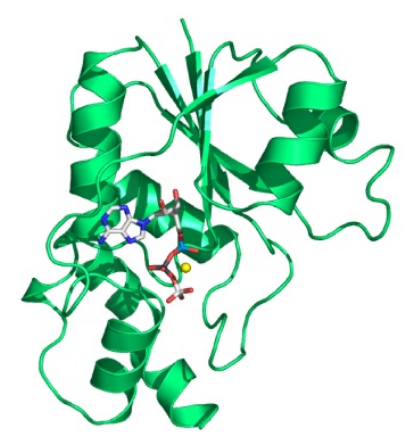

1f9a (ATP, $\left.\mathrm{Mg}^{2+}\right)$

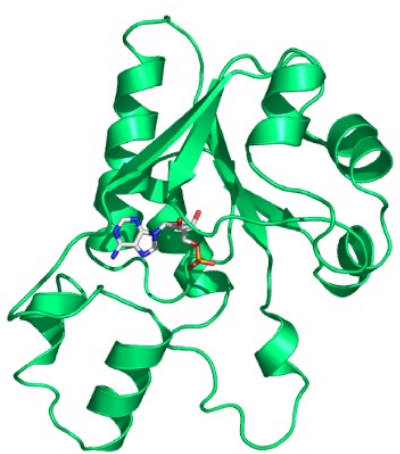

1t6y (AMP)

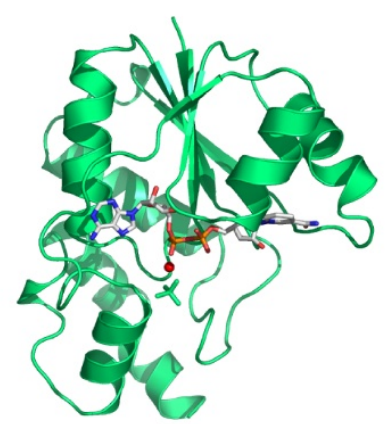

$1 \mathrm{ej} 2\left(\mathrm{NAD}^{+}, \mathrm{Na}^{+}, \mathrm{SO}_{4}{ }^{2-}\right)$

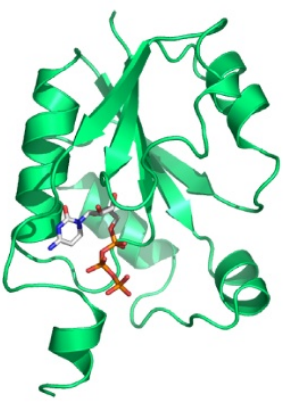

$1 \operatorname{coz}(\mathrm{CTP})$

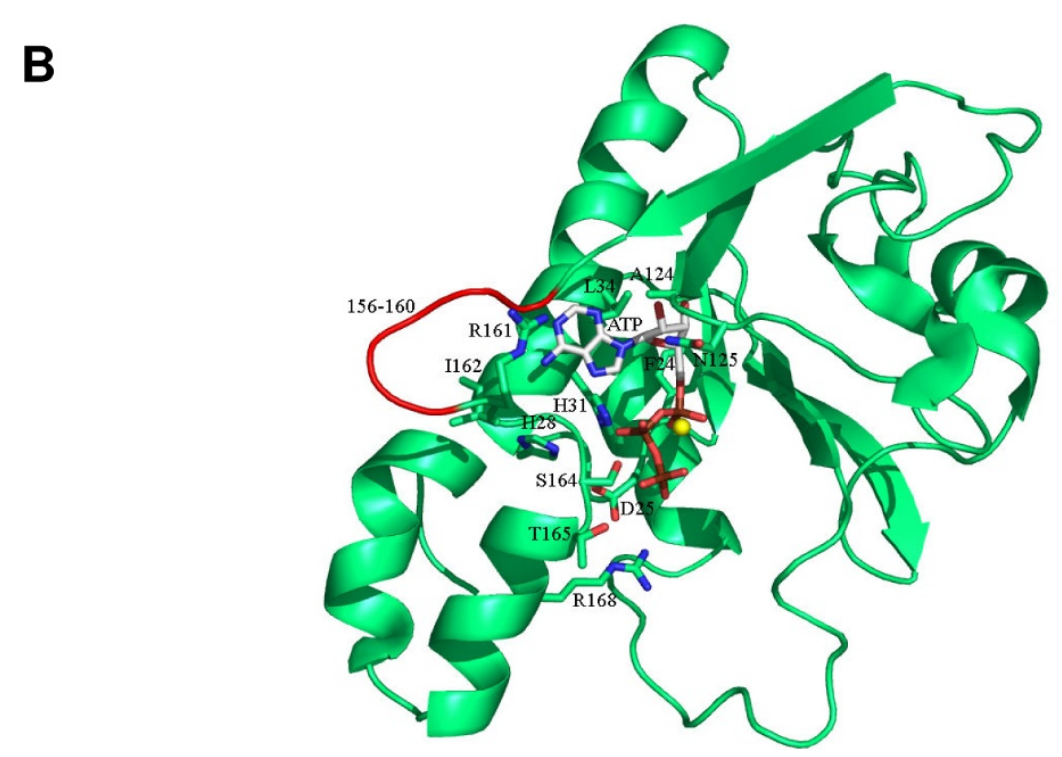

\section{Figure 5}

$\mathrm{N}$-terminal domain structure of CaFADS. (A) Comparison of the structural elements with those found in NTs. (B) Model for the interaction of ATP-Mg ${ }^{2+}$ with the N-terminal domain. Residues putatively involved in the NT activity and ATP binding are drawn as sticks and CPK coloured. Carbon atoms are shown in green and white, for the protein side-chains and nucleotides, respectively. $\mathrm{Mg}^{2+}$ and $\mathrm{Na}^{+}$ions are shown as yellow and red balls, respectively. 
A

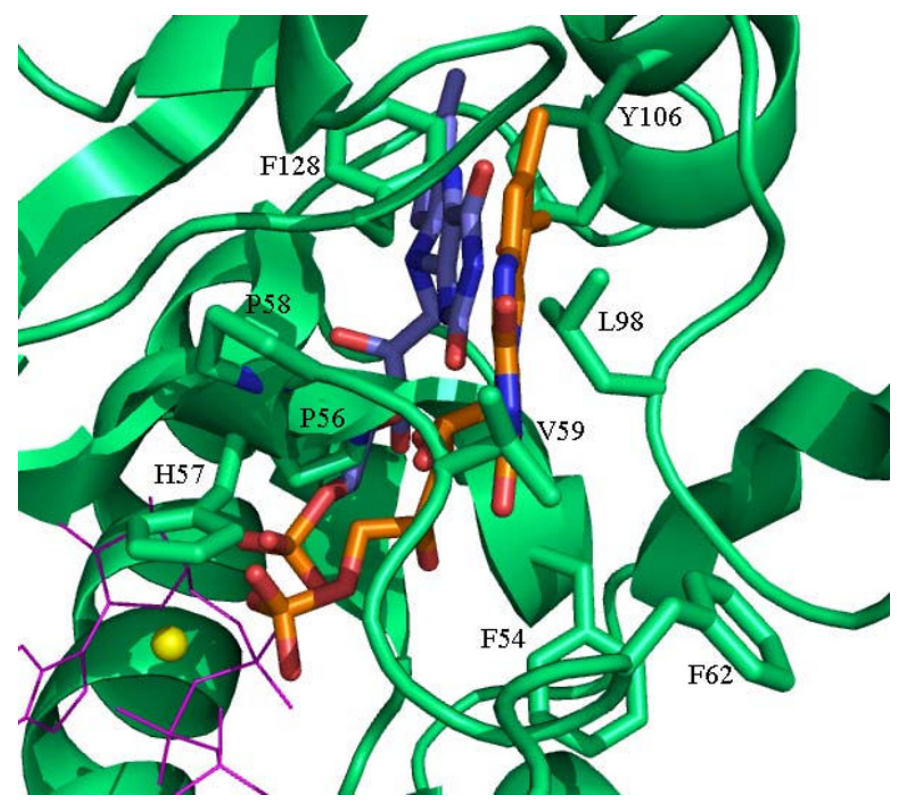

$\mathrm{B}$

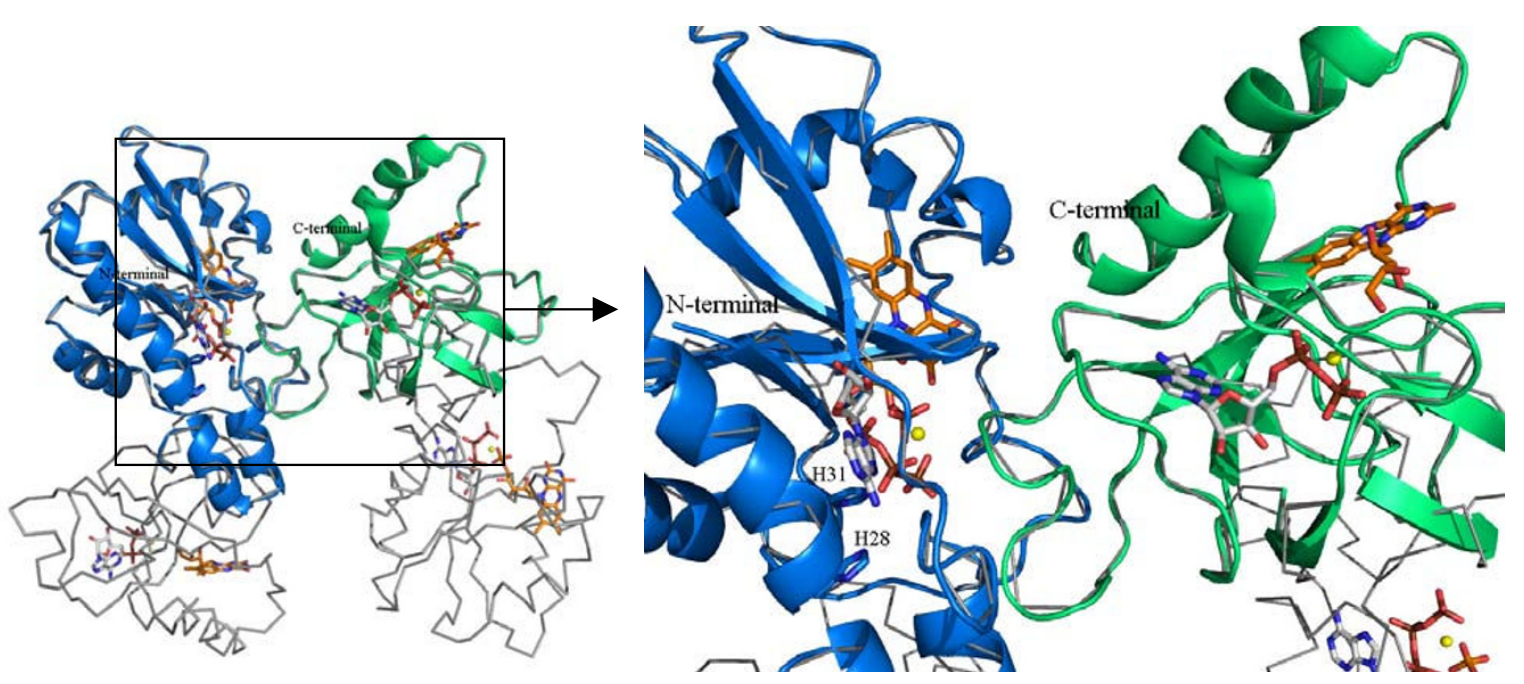

Figure 6

Localisation of the flavin binding site at the $\mathbf{N}$-terminal domain of CaFADS. (A) Models for the interaction of FMN with the $\mathrm{N}$-terminal domain. FMN and residues putatively involved in its stabilisation are drawn in sticks and CPK coloured. Two possible conformations for the FMN are indicated (Carbon atoms are in orange and blue, respectively). Positions of ATP and $\mathrm{Mg}^{2+}$ are also indicated as pink lines and a yellow ball, respectively. (B) Model for the interaction between two CaFADS monomers (grey lines), based on the TmFADS crystallographic dimeric crystal unit. The C-terminal of monomer I is shown in green and the N-terminal of monomer 2 in blue. Position of the RF and FMN substrates, as well as ATP-Mg' ${ }^{2+}$, are indicated at each binding site in CPK. Carbon atoms are shown in white and orange for nucleotides and flavins, respectively. $\mathrm{H} 28$ and $\mathrm{H} 3$ I are shown as sticks. 
lylation sites are far away (Figure 6B). This fact is in agreement with experimental observations indicating that the FMN produced in the phosphorylation process was released from the protein, to subsequently rebind to initiate the adenylylation process [9]. Superposition of our CaFADS model in complex with all its substrates with the dimer reported in the TmFADS crystallographic asymmetric unit also indicates that phosphorylation and adenylylation active sites from different monomers are far away to share the FMN molecule. However, this dimeric model suggests that the 229-243 loop and the terminal $\alpha 7$ of the C-terminal domain might contribute to the formation of the ATP and the flavin binding pockets in the adenylylation domain (Figure 6B).

\section{Production of C. ammoniagenes FAD synthetase forms} E. coli BL21(DE3) cells transformed with the recombinant PET28a-FADS vector produced a high level expression of active CaFADS. This CaFADS was purified in complex with one of the products of its reaction, FAD, as confirmed by HPLC (data not shown). To remove the FAD a hydrophobic chromatography was required. The sequence and structural analysis suggested the presence of several residues and motifs in CaFADS highly conserved in this family of enzymes and potentially involved in the Catalytic sites of the RFK and NT activities. Some of these residues were mutated, namely H28, H31, R161, S164, T165, T208, N210 and E268 to Ala and Asp. These CaFADS variants were expressed in E. coli, with yields similar to the WT FADS, and were purified to homogeneity following the same protocol. Additionally, plasmids individually expressing the $\mathrm{N}$-terminal and $\mathrm{C}$-terminal CaFADS domains were also produced, PET28a- $\Delta(183-338) F A D S$ and PET28a- $\Delta(1-182) F A D S$, respectively, and transformed in E. coli, showing a level of expression similar to the full length protein.

\section{C. ammoniagenes FAD synthetase residues involved in FMN and FAD production}

The ability of the different individual CaFADS mutants to catalyse conversion of RF into FMN and of FMN into FAD was analysed by incubating the same amount of each mutant with the substrates of both enzyme activities. Reactions were carried out under two different $\mathrm{Mg}^{2+}$ concentrations; one favouring the RFK activity $(0.8 \mathrm{mM})$ and the other favouring the NT activity $(10 \mathrm{mM})[7,11,12]$. However, both concentrations produced similar qualitative results under the assayed conditions. The products of the reactions were analysed by TLC (Figure 7).

Replacement of H28 with either Ala or Asp and H31 with Ala prevented conversion of FMN into FAD, confirming their implication in the CaFADS NT activity. Both of these His residues belong to a consensus region 27- $\Phi-\mathrm{H}-\Omega-\mathrm{G}-\mathrm{H}-$ $\Omega$-32 found both in FADS and NT families (Figure 1 and
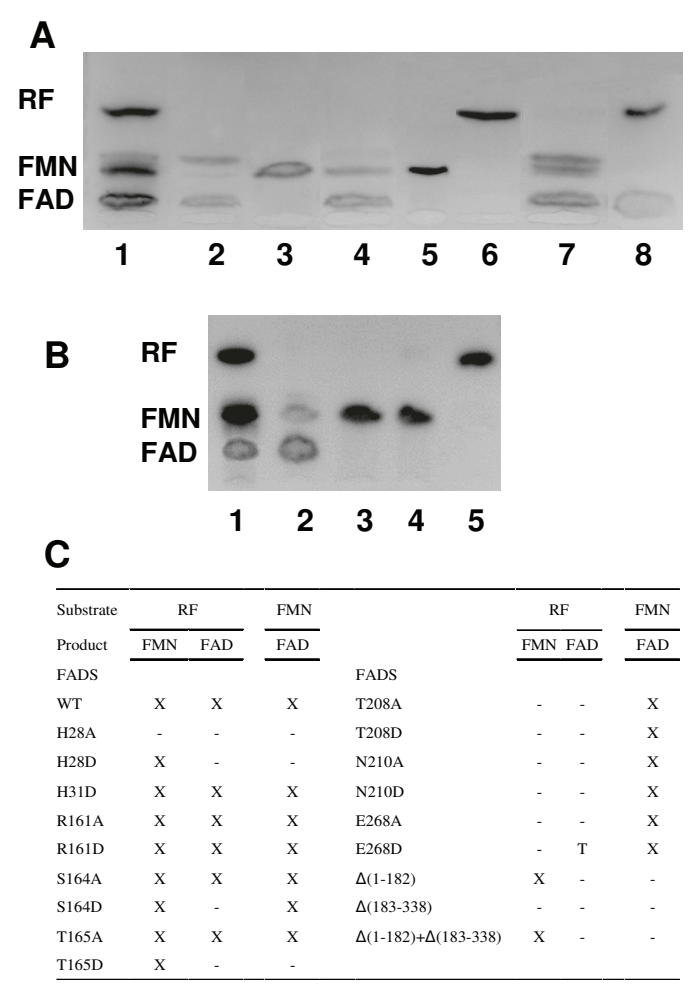

\section{Figure 7}

Resolution by thin layer chromatography of the products of the RFK and the NT activities of selected CaFADS variants. The reaction mixtures contained: (A), 2-WT and $50 \mu \mathrm{M} \mathrm{RF}, 3-\mathrm{H} 28 \mathrm{D}$ and $50 \mu \mathrm{M}$ RF, 4-RI6ID and $50 \mu \mathrm{M} \mathrm{RF}, 5-\mathrm{SI} 64 \mathrm{D}$ and $50 \mu \mathrm{M}$ RF, 6-T208A and $50 \mu \mathrm{M}$ RF, 7-T208A and $50 \mu \mathrm{M} F M N$ and 8-E268D and $50 \mu \mathrm{M}$ RF. (B), 2-WT CaFADS and $50 \mu \mathrm{M}$ RF, 3-CaFADS C-terminal domain and $50 \mu \mathrm{M} \mathrm{RF}, 4-C a F A D S \mathrm{C}$-terminal domain and $50 \mu \mathrm{M}$ FMN and 5-a reaction mixture containing $R F$ incubated in the absence of enzyme. Lanes ( $I$ ) in (A) and (B) corresponded to a standard solution containing $50 \mu \mathrm{M} R F, 50 \mu \mathrm{M}$ FMN and 50 $\mu M$ FAD. All the reaction mixtures contained $0.2 \mathrm{mM}$ ATP and $10 \mathrm{mM} \mathrm{MgCl}$ in $50 \mathrm{mM}$ Tris/ $\mathrm{HCl}, \mathrm{pH}$ 8.0. Experiments in (A) were carried out with pure CaFADS variants at concentration I $\mu \mathrm{M}$, while reaction mixtures in (B) contained crude extracts with the over-expressed CaFADS C-terminal domain. (C) Product formation in the assayed samples. $X, T$ and hyphen indicate production, detection of only traces and no production of the corresponding reaction product, respectively.

3A). Therefore, H28 and H31 will surely be major determinants in the stabilisation of the P groups and the adenine moiety of ATP in the reaction transition state in CaFADS, in agreement with previous observations in NTs $[31,32,34]$. Replacement of $\mathrm{H} 28$ with Ala also prevented the conversion of RF into FMN, pointing to a possible 
influence of the N-terminal domain in the RFK activity. His 28 appears situated at the $\mathrm{N}$-terminal of $\alpha 1$, in contact with $\alpha 5$ and $\alpha 6$, which, according to our model, will interact with the C-terminal domain from the same polypeptide chain, but also might influence the $\mathrm{C}$-terminal of the second monomer in a putative dimeric structure (Figure 6B). However, it is difficult to envisage how the H28A mutation might affect the phosphorylation reaction with the available information. This will need further structural and biochemical characterisation. Replacement of H31 with Asp produced an enzyme still able to produce FMN and FAD, suggesting that an Asp at this position somewhat replaces the function of the His. Replacements were also introduced in the second major consensus sequence shared by the FADS and the NT families, 162-ФSST-165. Replacement of S164 or T165 with Ala produced enzyme forms able to transform RF into FMN and FMN into FAD, while the introduction of a Asp residue at such positions clearly prevented FAD formation. This latter observation was consistent with these residues being involved in the stabilisation at the NT catalytic site of the negatively charged $\beta$-P and $\gamma$-P of ATP or the ribityl end, as suggested by the above structural analysis (Figures $3 \mathrm{~A}$ and $5 \mathrm{~B}$ ). Finally, the putative role of the structural disposition of the positive charge of R161 in the stabilisation of the adenine and $\alpha-P$ and $\beta$-P of ATP in the active site was studied by replacement of R161 with either Ala or Asp. The produced FADS mutants were still able to produce FMN and FAD under the assayed conditions, suggesting the positive charge at position of R161 is not playing a critical role in CaFADS catalysis.

Taking into account the fact that T208, N210, and E268 are conserved in sequence and structural position in the close environment of the ATP, not only in the FADS family but also in RFKs, and that the terminal oxygen of the ribityl of RF binding sites (Figures 1, 3B and 4), a catalytic role for them in the RF phosphorylation process can be envisaged. These CaFADS residues were replaced with Ala or Asp to prove such a role. All these mutations prevented FMN production, but allowed transformation of FMN into FAD, confirming their implication in the RFK activity of FADS. These data are, therefore, in agreement with the proposed structural-based role for T208 and N210 in accommodating the active portion of the ATP molecule, the $P$ groups, and the metal in the catalytic centre (Figure $4 B)$. The lack of FMN and FAD production when replacing E268 with Ala confirms it is a key residue in the CaFADS RFK activity. Analysis of the interaction of the CaFADS model with FMN (or RF) also indicated that in CaFADS E268 side-chain might $\mathrm{H}$-bond to the terminal $\mathrm{OH}$ of the ribityl chain of RF (Figure 4B). This Glu is invariant in the FADSs and RFKs families (Figures 1 and 3B). In this later family this residue has been proposed to act as a catalytic base [21-23]. Moreover, the fact that incubation of E268D with RF did not produce any accumulation of FMN, while traces of FAD were detected, is consistent with the RFK activity taking place at a very slow rate in this mutant, thus avoiding FMN accumulation. This suggests that although the introduced mutation is conservative and the Asp sidechain might still act as a base, its shorter side-chain prevents the optimal organisation of the transition state in the active centre and, therefore, reduces the catalytic efficiency of the RFK activity. Again, this is consistent with this residue not presenting substitutions, even conservative ones, in the analysed proteins. Nevertheless, other explanations might be also possible, as higher catalytic efficiency of NT activity than that of RFK or sensitivity of the TLC analysis. Further work should be done by the analysis of the kinetic parameters for both activities, as well as of the interaction parameters for the different substrates, for all these CaFADS mutants to provide additional insights in their specific role in the enzyme catalytic mechanisms.

\section{Activity of the independently produced $\mathrm{C}$. ammoniagenes FAD synthetase $\mathrm{N}$ - and $\mathrm{C}$ - terminal domains}

Crude extracts of the E. coli cells expressing the individually cloned $\mathrm{N}$-terminal and C-terminal domains of CaFADS, $\Delta(183-338)$ FADS and $\Delta(1-182)$ FADS, respectively, were prepared and assayed for NT and RFK activities (Figures 7B, 7C). Analysis of the products of the reaction by TLC concluded that the C-terminal domain, $\Delta(1-182) F A D S$, was able to qualitatively catalyse the phosphorylation process. However, crude extracts overexpressing the $\mathrm{N}$-terminal domain, $\Delta(183-338) F A D S$, were not able to catalyse the conversion of FMN into FAD. Extracts containing both domains were mixed and assayed for both reactions. Conversion of RF into FMN was produced in these samples, but they were not able to mimic CaFADS in the adenylylation process. This is in agreement with a possible C-terminal domain role in contributing to the stabilisation of the flavin and ATP substrates in the corresponding $\mathrm{N}$-terminal domain pockets (Figure 6B).

\section{Conclusion}

A sequence analysis has been carried out in the bifunctional FADS family. An important number of consensus regions and conserved residues have been determined, despite the low sequence identity among members of this family. Sequence divergences found among pathogenic organisms, such as Mycobacterium or Corynebacterium, and monofunctional eukaryotic enzymes will probably be reflected in structural divergences that might affect the catalytic mechanism, making possible the design of specific antimicrobial drugs. Models have been proposed, not only for the CaFADS structure, but also for the complexes formed with all its substrates. This includes de interaction with the FMN substrate at the adenylylation site, in a 
region not previously reported as involved in the enzyme activity and, in a pocket that might constitute a novel flavin binding motif. As expected, these models supported that the N-terminal presents similar folding to NTs and is involved in the adenylylation of FMN, whereas the C-terminal is similar to RFKs and responsible for the phosphorylation. Models of CaFADS allowed identification of several residues putatively involved in the catalysis. Sitedirected mutagenesis on some of these residues confirmed their participation in substrate stabilisation and/or the catalytic mechanism. The particular behaviour of some of these mutants and of the independently expressed NT and RFK domains suggests one of the domains might influence the activity in the other one. This study sheds important and new information on the structural mechanism of substrate recognition and catalysis while waiting for the experimental structure of the CaFADS and provides a platform for future investigations into the mechanism of enzymes of the bifunctional FADS family.

\section{Authors' contributions}

SF carried out the sequence alignment, contributed to production and analysis of the properties of WT FADS and some its mutants and drafted the manuscript. MM-J carried out the production of the three-dimensional FADS model structures free and in complex with ligands. AS participated in the production of the different FADS variants and in its characterisation. MM conceived of the study, and participated in its design and coordination, in the modelling of the three-dimensional structures and drafted the manuscript. All authors read and approved the final manuscript.

\section{Acknowledgements}

This work has been supported by the Spanish Ministry of Sciences (Grant BIO2007-65890-C02-0I to M.M.).

\section{References}

I. Barile M, Brizio C, Valenti D, De Virgilio C, Passarella S: The riboflavin/FAD cycle in rat liver mitochondria. Eur J Biochem 2000, 267(15):4888-4900.

2. McCormick DB, Oka M, Bowers-Komro DM, Yamada Y, Hartman HA: Purification and properties of FAD synthetase from liver. Methods Enzymol 1997, 280:407-4I3.

3. Oka M, McCormick DB: Complete purification and general characterization of FAD synthetase from rat liver. J Biol Chem 1987, 262(15):7418-7422.

4. Merrill AH Jr, McCormick DB: Affinity chromatographic purification and properties of flavokinase (ATP:riboflavin 5 '-phosphotransferase) from rat liver. I Biol Chem 1980, 255(4): I335-1338.

5. Brizio C, Galluccio M, Wait R, Torchetti EM, Bafunno V, Accardi R, Gianazza E, Indiveri C, Barile M: Over-expression in Escherichia coli and characterization of two recombinant isoforms of human FAD synthetase. Biochem Biophys Res Commun 2006, 344(3): $1008-1016$.

6. Galluccio M, Brizio C, Torchetti EM, Ferranti P, Gianazza E, Indiveri C, Barile M: Over-expression in Escherichia coli, purification and characterization of isoform 2 of human FAD synthetase. Protein Expr Purif 2007, 52(1): I75-181.
7. Manstein DJ, Pai EF: Purification and characterization of FAD synthetase from Brevibacterium ammoniagenes. I Biol Chem |986, 26 I(34):16169-16173.

8. Grill S, Busenbender S, Pfeiffer M, Kohler U, Mack M: The bifunctional flavokinase/flavin adenine dinucleotide synthetase from Streptomyces davawensis produces inactive flavin cofactors and is not involved in resistance to the antibiotic roseoflavin. J Bacteriol 2008, I 90(5): I546-I553.

9. Efimov I, Kuusk V, Zhang X, Mclntire WS: Proposed steady-state kinetic mechanism for Corynebacterium ammoniagenes FAD synthetase produced by Escherichia coli. Biochemistry 1998, 37(27):9716-9723.

10. Murthy YV, Massey V: Syntheses and applications of flavin analogs as active site probes for flavoproteins. Methods Enzymol 1997, 280:436-460.

II. Hagihara T, Fujio T, Aisaka K: Cloning of FAD synthetase gene from Corynebacterium ammoniagenes and its application to FAD and FMN production. Appl Microbiol Biotechnol 1995, 42(5):724-729.

12. Nakagawa S, Igarashi A, Ohta T, Hagihara T, Fujio T, Aisaka K: Nucleotide sequence of the FAD synthetase gene from Corynebacterium ammoniagenes and its expression in Escherichia coli. Biosci Biotechnol Biochem 1995, 59(4):694-702.

13. Krupa A, Sandhya K, Srinivasan N, Jonnalagadda S: A conserved domain in prokaryotic bifunctional FAD synthetases can potentially catalyze nucleotide transfer. Trends Biochem $\mathrm{Sci}$ 2003, 28(I):9-12.

14. Wang W, Kim R, Jancarik J, Yokota H, Kim SH: Crystal structure of a flavin-binding protein from Thermotoga maritima. Proteins 2003, 52(4):633-635.

15. Wang W, Kim R, Yokota H, Kim SH: Crystal structure of flavin binding to FAD synthetase of Thermotoga maritima. Proteins 2005, 58(I):246-248.

16. Altschul SF, Madden TL, Schaffer AA, Zhang J, Zhang Z, Miller W, Lipman DJ: Gapped BLAST and PSI-BLAST: a new generation of protein database search programs. Nucleic Acids Res 1997, 25(I 7):3389-3402.

17. Thompson JD, Higgins DG, Gibson TJ: CLUSTAL W: improving the sensitivity of progressive multiple sequence alignment through sequence weighting, position-specific gap penalties and weight matrix choice. Nucleic Acids Res 1994, 22(22):4673-4680.

18. Crooks GE, Hon G, Chandonia JM, Brenner SE: WebLogo: a sequence logo generator. Genome Res 2004, I 4(6): I I 88-I I 90.

19. Combet C, Jambon M, Deleage G, Geourjon C: Geno3D: automatic comparative molecular modelling of protein. Bioinformatics 2002, I 8(I):2|3-2|4.

20. Laskowski RA, MacArthur MW, Moss DS, Thornton JM: PROCHECK: a program to check the stereochemical quality of protein structures. J Appl Cryst 1993, 26:283-291.

21. Karthikeyan S, Zhou Q, Mseeh F, Grishin NV, Osterman AL, Zhang $\mathrm{H}$ : Crystal structure of human riboflavin kinase reveals a beta barrel fold and a novel active site arch. Structure 2003, II (3):265-273

22. Karthikeyan S, Zhou Q, Osterman AL, Zhang H: Ligand bindinginduced conformational changes in riboflavin kinase: structural basis for the ordered mechanism. Biochemistry 2003, 42(43): $12532-12538$

23. Bauer S, Kemter K, Bacher A, Huber R, Fischer M, Steinbacher S: Crystal structure of Schizosaccharomyces pombe riboflavin kinase reveals a novel ATP and riboflavin-binding fold. J Mol Biol 2003, 326(5): | 463-1473.

24. Jones TA, Zou JY, Cowan SW, Kjeldgaard M: Improved methods for building protein models in electron density maps and the location of errors in these models. Acta Crystallogr A I991, 47(Pt 2): $110-119$

25. Guex N, Peitsch MC: SWISS-MODEL and the Swiss-PdbViewer: an environment for comparative protein modeling. Electrophoresis 1997, 18( I 5):27|4-2723.

26. Delano WL: They PyMOL molecular graphics system. 2002 [http://www.pymol.org]. DeLano Scientific, San Carlos, CA, USA

27. Pospiech A, Neumann B: A versatile quick-prep of genomic DNA from Gram-positive bacteria. Trends in Genetics 1995, II (6):2I7-2I8.

28. Bauer K, Gros L, Sauer W: Thin Layer Chromatography: An Introduction. Germany: Hüthig Verlag; 1991. 
29. Berg PAW van den, Feenstra KA, Mark AE, Berendsen HJC, Visser AJWG: Dynamic conformations of flavin adenine dinucleotide: Simulated molecular dynamics of the flavin cofactor related to the time-resolved fluorescence characteristics. J Phys Chem B 2002, I06(34):8858-8869.

30. Weber CH, Park YS, Sanker S, Kent C, Ludwig ML: A prototypical cytidylyltransferase: CTP:glycerol-3-phosphate cytidylyltransferase from Bacillus subtilis. Structure 1999, 7(9): I II3-II 24

31. Garavaglia S, D'Angelo I, Emanuelli M, Carnevali F, Pierella F, Magni G Rizzi M: Structure of human NMN adenylyltransferase. A key nuclear enzyme for NAD ${ }^{+}$homeostasis. I Biol Chem 2002, 277(10):8524-8530.

32. D'Angelo I, Raffaelli N, Dabusti V, Lorenzi T, Magni G, Rizzi M: Structure of nicotinamide mononucleotide adenylyltransferase: a key enzyme in NAD+ biosynthesis. Structure 2000, 8(9):993-1004.

33. Saridakis V, Christendat D, Kimber MS, Dharamsi A, Edwards AM, Pai $\mathrm{EF}$ : Insights into ligand binding and catalysis of a central step in NAD ${ }^{+}$synthesis: structures of Methanobacterium thermoautotrophicum NMN adenylyltransferase complexes. J Biol Chem 200I, 276(10):7225-7232.

34. Izard T, Geerlof A: The crystal structure of a novel bacterial adenylyltransferase reveals half of sites reactivity. Embo J I999, I 8(8):202 I-2030.

35. Takahashi H, Inagaki E, Fujimoto $Y$, Kuroishi C, Nodake $Y$, Nakamura Y, Arisaka F, Yutani K, Kuramitsu S, Yokoyama S, et al.: Structure and implications for the thermal stability of phosphopantetheine adenylyltransferase from Thermus thermophilus. Acta Crystallogr D Biol Crystallogr 2004, 60(Pt I):97-I04.

36. Mizuguchi KDC, Blundell TL, Johnson MS, Overington JP: JOY: protein sequence-structure representation and analysis. Bioinformatics 1998, 14:617-623.

37. Izard $\mathrm{T}$ : The crystal structures of phosphopantetheine adenylyltransferase with bound substrates reveal the enzyme's catalytic mechanism. J Mol Biol 2002, 3 I 5(4):487-495.

Publish with Bio Med Central and every scientist can read your work free of charge

"BioMed Central will be the most significant development for disseminating the results of biomedical research in our lifetime. "

Sir Paul Nurse, Cancer Research UK

Your research papers will be:

- available free of charge to the entire biomedical community

- peer reviewed and published immediately upon acceptance

- cited in PubMed and archived on PubMed Central

- yours - you keep the copyright
BioMedcentral 